\title{
Digital Affordances and Evaluative Stance: Redefining Hard News
} Orbital Structure

\section{Rania Magdi Fawzy}

\author{
Associate Professor of Applied Linguistics at \\ the College of Language \& Communication (CLC), Arab Academy for \\ Science, Technology and Maritime Transport (AASTMT), Heliopolis Branch. \\ E-Mail: raniamagdi@aast.edu
}

\section{ABSTRACT}

This study approaches the question of how text generic structure acts as semiotic instantiations of evaluative stance, particularly in the online context. In this respect, it explores the generic structure of online reportage stories with its multitude modes, distinctive rhetorical structure and layout with a view to differentiating them from their printed counterparts and extending the theoretical tools in this area by introducing slight modifications to ledema, Feez and White's (1994) model for hard news generic structure. Doing so, the study employs Mann and Thompson's (1988) approach to genre so as to determine reportage nuclearity and Thompson and Hunston's (2000) model of evaluation to relate evaluation to text organisational structure. It takes a case study of one of New York Times online reportage stories criticising Egypt's sugar crisis (2017) to trace how the schematic structure of this story influences and multiplies the evaluative stance. The delicate modifications intend to help in digging deep into the embedded evaluative stance of the online news reportage stories. The study concludes that online reportage can be recognized as a macro genre that includes two interrelated yet independent subgenres with two nucleus-satellites unites. As for the underlying evaluative stance, the analysis reveals how the NYT reportage story under discussion evaluates the Egyptian sugar crisis along with negatively attributing it to the current government.

Keywords: Digital affordance; Evaluative stance; Online genre; Orbital structure; Reportage stories

\section{PRELUDE}

Many linguists examine genres for their social functions (Bateman 2008; Bhatia 1993; Lemke 1998; Martin 2013; Miller 1994; Swales 1990). They highlight the role played by genre in the construction of social realities and communicative purposes. Informed by such considerable body of research on genre and its social role, this paper examines the role of online genre in instantiating evaluative stance. It questions how the tone of evaluation is related to the structure of online reportage stories. In this respect, it explores the generic structure of online reportage with its multitude modes, distinctive rhetorical structure and layout with a view to differentiating them from their printed counterparts.

The paper takes a case study of one of New York Times (NYT) online reportage stories covering the Egyptian sugar crisis (2017). It examines how the reportage evaluative stance is constructed through the combination of various semiotic resources in the non-verbal (images, hyperlinks and text typography) and in the verbal (text) modes to examine the impact of digital affordances on reportage stories generic structure.

\section{ORBITAL STRUCTURE SCHEME: Some Reflections on Digitized News}

Researchers explore the intersemiotic modes underlying multimodal texts (Caple 2009, 2008a,b; Kourdis and Yoka 2014; Kress 2009, 2010; Lemek 1998, Royce 2007; Wignell, O'Halloran and Tan 2016). Serafini $(2010,87)$ concludes that multimodal texts present information across a variety of modes including visual 
images, design elements, written language, and other semiotic resources. Focusing on the genre of digital multimodality, Deuze $(2008,209)$ calls for a rigorous investigation of the practice of online journalism with its numerous new formats, genres and innovations in the production of online news. In their work Multimodal Transcription and text analysis, Baldry and Thibault (2006) examine whether the notion of genre can be applied to hypertexts. They find that websites have generic structures of their own, yet they function through the interplay of various modes. Hypertext, according to them, is a "hybrid of precursor genres such as verbal text, visual images, and multimodal combinations of these" (Baldry and Thibault 2006, 156 italics in the original).

In the hard news story, genre events are nuclearized and logical relations are disrupted (ledema et al. 1994; White 1997). The laying out of events leading up to the crisis point becomes more optional in the story, meaning that causation and temporality become less important organizing principles. Rather, the news story now centres on a crisis point established in the headline and the lead (the nucleus), which then becomes the platform from which readers leap into the remainder of the story (Caple 2009, 254).

ledema et al. (1994), White (1997) and Feez, ledema and White (2008) suggest orbital schematic structure which interrelates the functionality of text component parts. Orbital organization is the opposite of the linear one in the sense that text elements are built in what comes immediately before or prepare the way for what is to follow immediately after rather than linking together or build a linear semantic 'pathway' through which meaning is built up serially (White 1997, 115).

They propose a Nucleus ^ Satellite orbital model. Nucleus stands for the basis for how the story will develop. Meanwhile, satellites reformulate the information given in the nucleus (ledema et al. 1994). This schematic organization contradicts the fixed linear organization. It examines the functional structure of the hard news story in which "dependent 'satellites' elaborate, explain, contextualise and appraise a textually dominant 'nucleus'" (ledema et al. 1994; White 1997, 111). In other words, they offer a schematic structure that does not link together to establish a linear reading pathway by which meaning is built up in terms of importance, rather "it reaches back to the headline/lead nucleus, which acts as the text's anchor point or textual centre of gravity" (White 1997, 115). This notion is illustrated in the following figure:

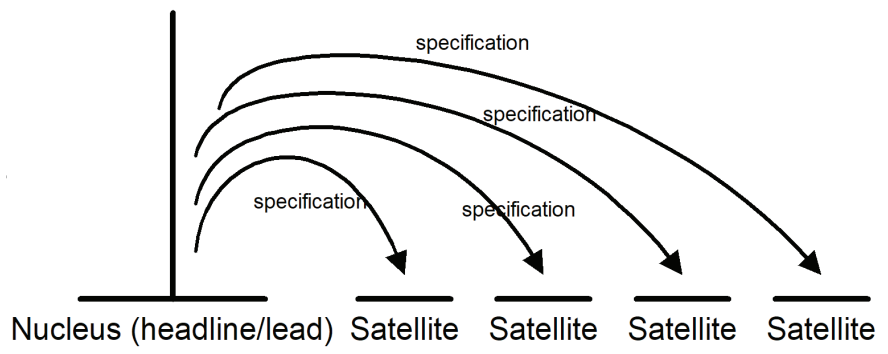

Figure 1. Orbital structure as proposed by White (1997)

This figure demonstrates that the main role of the body text is "not to develop new meanings but rather to refer back to the Headline/Lead through a series of specifications" (White 1997, p.115). Concerning news story orbital structure, ledema et al. (1994) comment on the function of the nucleus as telling the entire story and, thus, achieving the text social function. Nuclearity then orients reader's response to the text (Mann \& Thompson 1992, 271).

Thus, nuclearity, as stated by the previous two definitions, presents the core of the news story that contains the main evaluative resources, which, in turn, aligns the readers to the text according to the writer's intention. Following the nucleus, satellites function as an elaboration and extension of what is mentioned in the headline/lead in a variety of ways. The following figure illustrates the Nucleus ^ Satellite structure as put forward by ledema.

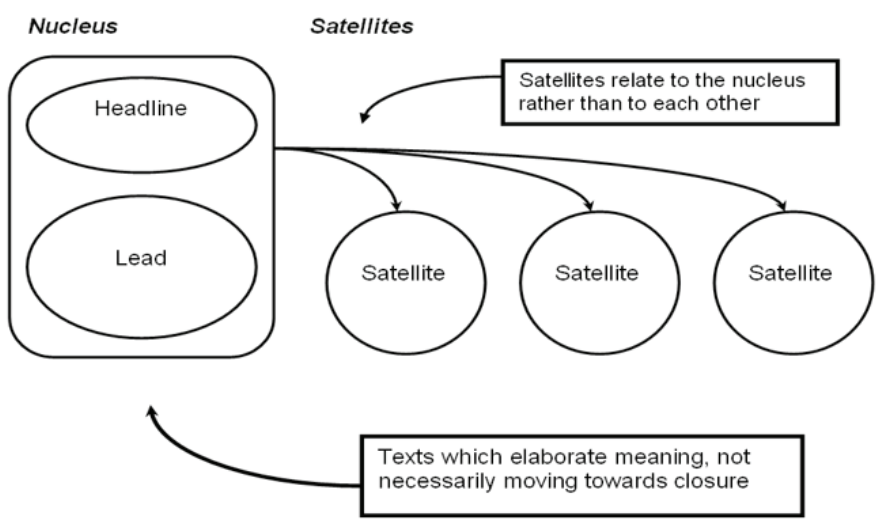

Figure 2. Nucleus ^ ${ }^{\wedge}$ Satellite structure 


\section{EVALUATION AND GENRE}

Evaluation defines the speaker or writer's attitude or stance towards the propositions that he or she is talking about (Thompson and Hunston 2000, 5). Text generic structure influences the underlying evaluative meanings (Bolívar 2001, 146; Coffin and O'Halloran 2005; Thompson ans Hunston 2000). As Thompson and Hunston $(2000,19)$ put it "evaluation tends to be found throughout a text rather than being confined to one particular part of it". They argue that that evaluation is even identified according to its position in a text and the role that it plays according to that position. Following the same line, Coffin and O'Halloran $(2005,144)$ investigate the accumulation of attitudinal meanings in the process of reading and define it as 'evaluative groove'. Evaluative groove, according to them, is "the way in which interpersonal meaning through a text can dynamically channel readers to take up an overall evaluative stance towards the content of subsequent text".

The concept of evaluative groove is referred to by (Martin and White 2005, 32), though with different terminology, within the discipline of Appraisal. From an Appraisal perspective, genre is seen as a "staged, goal oriented social process", since "we participate in genres with other people [...] to get things done [...] because it usually takes us a few steps to reach our goals". Correspondingly, Appraisal theory considers the evaluative strategies that are generically expressed to achieve such goals, and how these strategies evolve 'dynamically' from one stage of a given genre to another. Martin and White $(2005,33)$ stress "from the perspective of appraisal, we are interested in the range of evaluations the genre draws on to achieve its goals and how it plays out these evaluations from one stage of the genre to another".

\section{METHODOLOGY AND THEORETICAL FRAMEWORK}

The study at hand examines the notions of both evaluation and online multimedia mainly from a genre sensitive approach. It approaches the question of how evaluation is related to the structure of a given text. So doing, the study explores the generic structure

Figure 3. The schematic structure of online news reportage as displayed in NYT webpage of an online reportage story and relates it to the embedded evaluative stance. The concept of evaluation is then related to the verbal, visual and hypertextual properties of the online domain. Doing so, the study employs Thompson and Hunston's (2000) approach to text evaluation to investigate the evaluative stance embedded in New York Times (NYT) reportage under discussion. According to them, evaluation is achieved through lexis (adjectives, adverbs, nouns and verbs), grammar (intensifiers, comparators, correlatives and explicatives) and text organisational structure. However, the current study extends these suggested parameters for analysing evaluation to include visual cues as well.

The study deploys as well Mann and Thompson's (1988) approach to genre so as to determine reportage nuclearity. Moreover, the exploration of online reportage generic structure proposed in the study is drawn on ledema's et al. (1994) and White's (1997) suggested Nucleus ^ Satellite structure. The paper takes a case study of one of NYT online reportage stories covering the recent Egyptian sugar crisis (2017).

\section{DISCUSSION AND FINDINGS}

\section{The Generic Structure of Online Reportage Stories: An Evaluative Take}

The paper at hand examines how online reportage can be recognized as a macro genre that includes two interrelated yet independent sub-genres. It is significant to underline first the compositional elements of online features, consider the following figure:

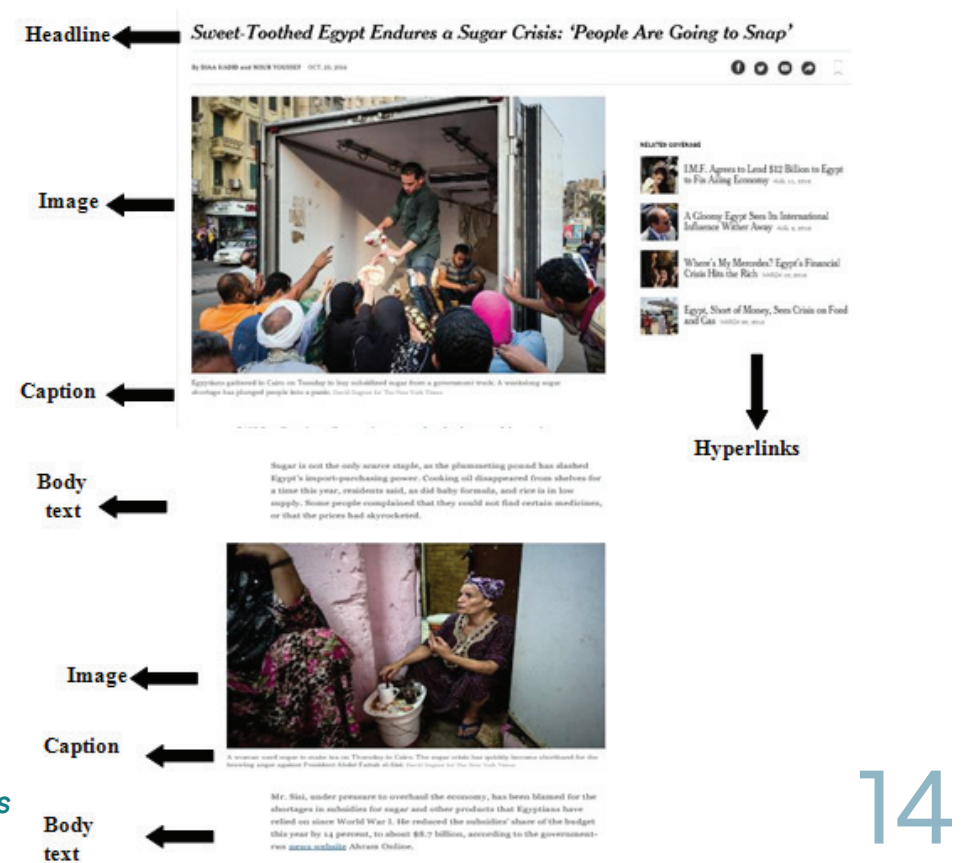


Every reportage story of the NYT, as displayed in Figure 3, provides a text of the article, positioned in the centre of the page and preceded by an image which is accompanied by a headline and a caption. Most of the time, it includes as well more than one image with their captions within the text itself. All these elements come with interactive hyperlinks of related news stories.

Figure 3 demonstrates that online reportage stories have the benefit of the digital affordances; combining visual and hypertextual elements which are not offered to the print ones. In this regard, designing a schematic structure that examines the visual and digital affordances of online reportage and how they are related to the accompanied verbal information should be considered. From this particular point, assigning text nuclearity is considerably essential.

The importance of determining nuclearity before assigning suitable schematic structure is best expressed in the words of Mann and Thompson (1988, 38), "nuclearity is thus an expressive resource that directs the reader to respond to the text in a particular and locally structured way. It seems to strongly influence the overall response that the writer intended". Hence, determining nuclearity helps trace the functionality of the text composing elements as well as its evaluative stance. Allocating the nuclear-satellites unit highlights the intersemiotic relations among the composing elements of multimodal texts as well as signalling their evaluative stance.

Given Kress and van Leeuwen's (2006) statement concerning the 'fall of 'monomodality' over the past century, it would be beneficial to question the notion of 'monouclearity' in the analysis of online reportage. Based on the assumption that NYT reportage stories include both verbal, visual and hypertextual elements, the present study proposes that online reportage stories are binuclear; with central two separate textual nuclei (the headline/ image) and the (lead), dominating a set of textual satellites acting to specify and elaborate these two nuclei. As such a reworking of White's (1997) proposed orbital structure is required:

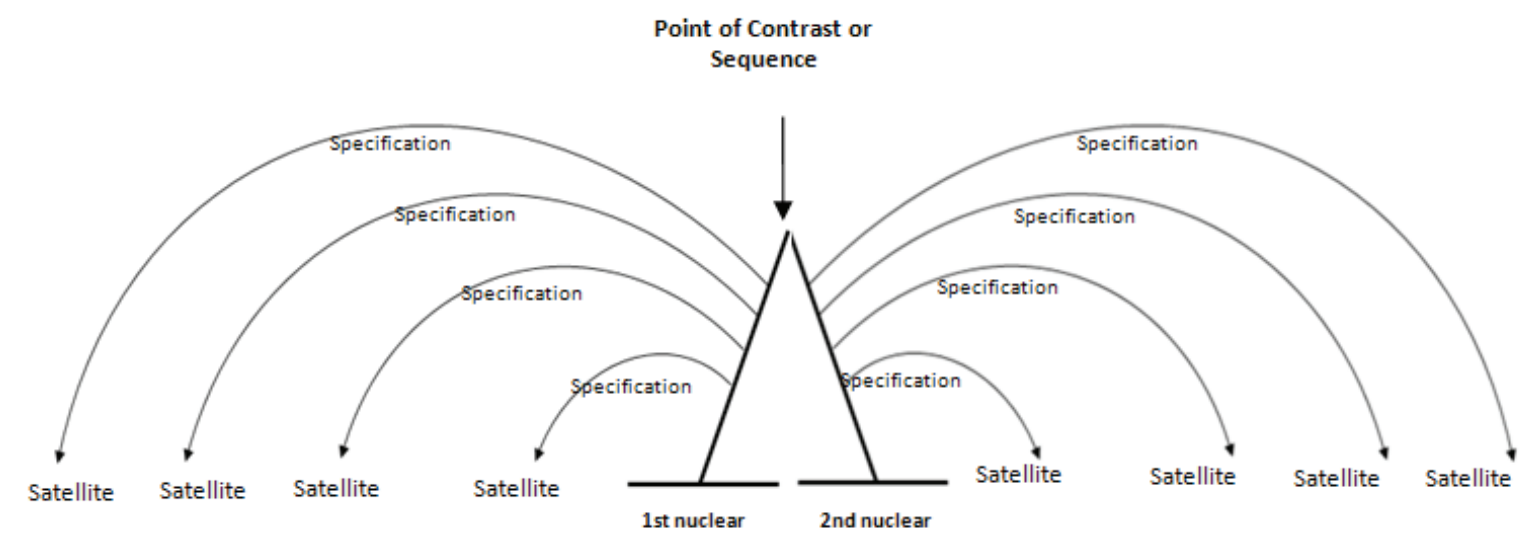

Figure 4. Proposed multinuclear structure as adopted from White's (1997)

The proposed figure marks the possibility of including two nuclei in a text that are met at some point of text evaluation. Correspondingly, the current paper finds that NYT online reportages can be considered as a binuclear text where the first nuclear-satellite units consist of elements that have both visual and verbal properties; due to typography: images, headline, caption and hyperlinks. Images, although being a visual element, they may include verbal quotes. As for the headline and caption, they afford typographic and layout visual resources. Hyperlinks are considered to be verbal and non-verbal elements due to the visual affordances of clickable items; blue coloured font, for instance. Concerning the second nuclear-satellite unit, it consists of the verbal text of the reportage (lead and lead developments) and the in-text hyperlinks. Informed by ledema's et al. (1994), the paper adapted their suggested orbital structure in assigning the schematic structure of print hard news stories.

However, in assigning the nucleus and satellites of the reportage under discussion, the researcher follows the test proposed by Mann and Thompson (1988). This test determines and differentiates the nucleus and satellites parts according to their function "if units that only function as satellites and never as nuclei are deleted, the text message is still there while [...] if the units that function as nuclei anywhere in the text are deleted, the 
main message would be difficult to convey." Therefore, the nucleus is the unit where the theme and significance lay.

Following Mann and Thompson's (1988) same line, nuclearity of the reportage analyzed can be tested with the deletion test. Applying such test, it is concluded that the first nucleus-satellite unit consists of an image, headline, caption, subsequent images and related news hyperlinks. It is then suggested that the image and the headline function as the nucleus; whereas the other structural elements; caption, related news hyperlinks and subsequent images can be considered as satellites.

The headline of Figure 3 helps orient the readers to the writer's negative stance against the economic crisis inflicting Egypt.

\section{Sweet-Toothed Egypt Endures a Sugar Crisis: 'People Are Going to Snap'}

The proposition of the headline is monoglossically formulated. The headline contains a strong trace of authorial intervention in the form of the idiomatic adjective 'sweet-toothed' and could be read as an authorial summary of the economic environment in Egypt. Monoglossic alignment between writer and reader is then construed via the foregrounding of the writer's negative stance towards the Egyptian political state of affairs after the economic crisis. Hence, the authorial voice resorts to idiomatic sharpness (Chenguang 2007) to reinforce the proposed negative evaluation. 'Sweet-toothed' means to desire to eat many sweet foods-especially candy and pastries.

The phrase voices an authorial opinion which has some negative connotations. Therefore, the headline functions interpersonally as the nucleus of evaluation. Intersemiotically, the image takes the role of the 'identifier' (Martinec and Salway 2005); it visualizes the verbal stance of the headline as represented in the depiction of a crowd of people standing before a vehicle distributing staples. The participants' outstretched arms, the most salient visual element due to visual repetition, symbolise their needs. A multiplication of the negative stance voiced against the Egyptian economic status is, then, achieved.

The caption then contextualizes the picture by naming the place: Cairo, and the time medium: weeklong of the sugar crisis. The chosen lexical items 'weeklong' and 'sugar crisis' further enhance the stance expressed in the suggested nucleus unit. However, the caption alone can be read as factual statement expressed in the 'reporter voice' (Martin and White, 2005) and, therefore, its evaluation is only achieved by accompanying the image.

The study considers as well the inclusion of hyperlinks to the first visual-verbal nucleus unit. Print reportage combines the elements of headline, photo, and caption in their schematic structure. In this sense, online reportage with the affordances of new technology should benefit from the online domain to be differentiated from the print ones. Although it is not found in the literature, to the best of the author's knowledge, the study has found it significant to include related news hyperlinks to the first nucleus unit as satellite.

Related news hyperlinks are termed by Knox (2009) as 'newsbites'. The following figure provides an illustration of related news compositional parts:

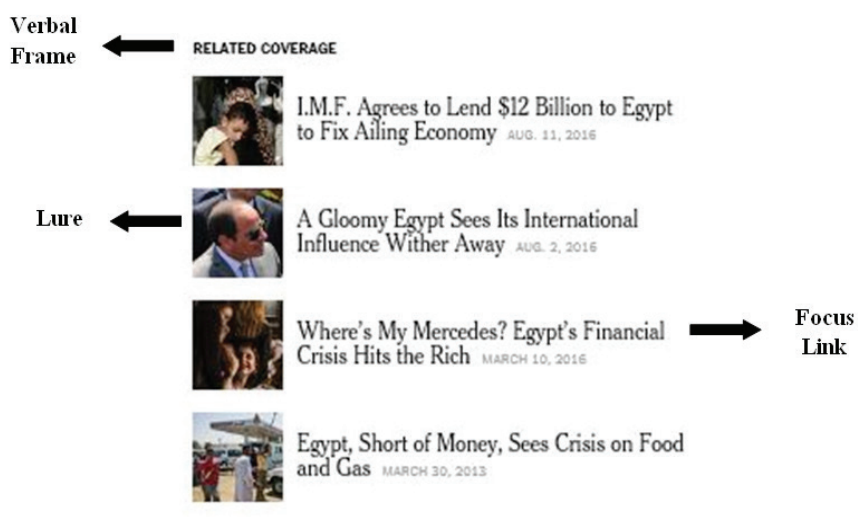

Figure 5. Related stories with all structural elements indicated

As illustrated by Figure 5, the related stories newsbites consist of a 'Verbal Frame', a 'Focus Link', and a 'Lure' (Knox 2009, 330). As its name suggests, the Verbal Frame relates the followed hyperlinks to the accompanied reportage. As for the Focus Link, it takes the form of a headline that is conflated with a link to a story page. In this regard, it has an intertextual function. Studying the reportage under discussion, it is found that the wording and lexical choices of the Focus Links move towards a negative evaluative stance against the Egyptian economic status. The lexical items 'ailing economy', 'gloomy Egypt', 'financial crisis' and 'short of money', essentially relate to and multiply the voiced 
negative evaluation against Egypt's economy. In this regard, the Focus Link stands for expanding dialogism (Tan 2008) since it invites to a longer version of the same story. On the left side of the Focus Link there is a hyper image. It visualizes the verbiage of the Focus consequently; it is given the functional label of 'Lure' (Knox 2009, 327).

The visual design of the reportage accompanied newsbites takes the form of the visual discourse of traditional news genres. The reportage newsbites relate to a longer version of the same story which has the same headline. Significantly, related stories newsbites are depended stories that point to longer news stories of various genres (editorials, articles, features etc.) elsewhere in the newspaper. This special feature of the story reveals how various voices are represented by what Fairclough $(1995,77)$ terms "Intertextual chain relations." In this sense, related news stories can be perceived as a technique of 'mixed genres' since they are intertxtually related to the adjunct feature article.

Based on the assumption that online newspapers provide an accessible source of authentic, target language material, Djonov, Knox and Zhao $(2015,335)$ propose some questions that are useful for teachers and learners of second and foreign languages concerning related news hyperlinks. Although proposed in a different context, these questions prove to be relevant to this part of the analysis which proposes the significance of linking these related stories newsbites with the nucleussatellite unit. These questions are:

How is the page designed? What visual and verbal framing devices are used to categorise content? Which stories are grouped together and how is this achieved visually? Are borders, frames, white space, and \or headings used? How do these devices work to create explicit or implicit categorization of the news? (emphasis added)

Therefore, these questions significantly examine the function of newsbites since they indicate that their placement adjunct to the news reports is not accidental, rather they are placed there for an ideological reason.
As satellites, they function as evaluative framing devices which reflect the position of the news organization towards the reportage story under discussion by relating them ideologically to other stories that are 'related', as claimed by the editorial board. Hence, these hyperlinks are placed beside the feature story for a reason. They expand the negative framing provided by the nucleus unit.

In this regard, it is assumed that NYT online reportage stories can be considered a macro genre which consists of two subgenres organized in orbital structure. The first sub-genre is constituted of picture, headline, caption, and related stories hyperlinks where the image and headline together form the nucleus part of evaluation and the other elements are the satellites which significantly expand the evaluative tone. The second sub-genre is the story body text.

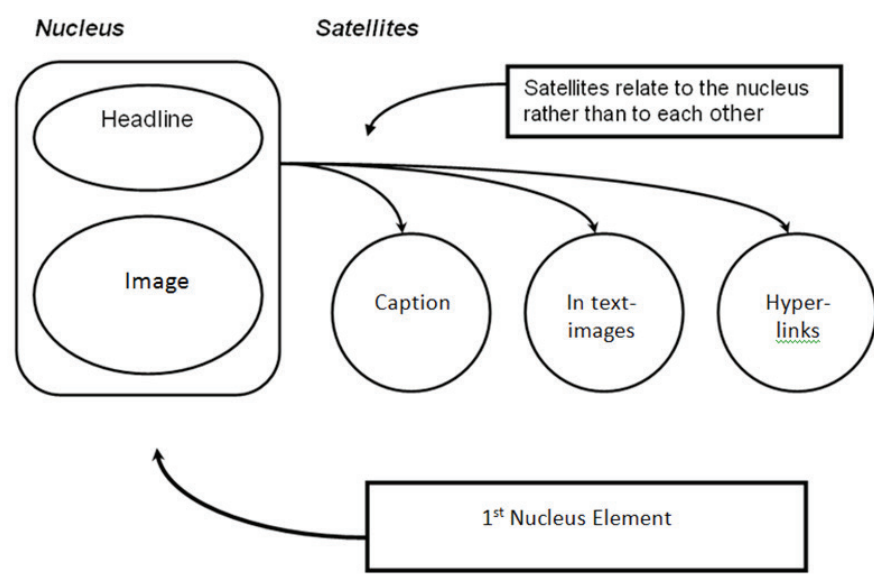

Figure 6. Proposed modifications on ledema's et al (1994) to fit into the online domain

\section{DETERMINING THE GENERIC STRUCTURE OF THE SECOND NUCLEAR-SATELLITE UNIT}

The second nucleus-satellite elements of NYT online reportage deal with the body text. The upright pyramid structure is the traditionally established schematic structure for printed reportage. Figure 7 is illustrative:

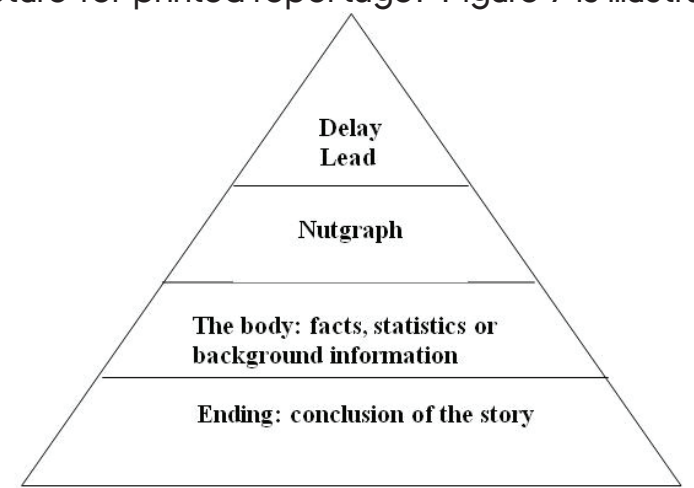

Figure 7. Upright pyramid (Johnston, 2007) 
The delayed lead hints at the story content in a brief narrative or descriptive form. It is then followed by a nut graph. As the figure displays, the upright pyramid structure places the real climax or features of a story somewhere other than in the lead. This technique is known as the 'suspended interest story' which provides excitement about the story. Often the climax in a story is in the final paragraph (Johnston 2007). It is usually presented by the second paragraph following the delay lead. However, such schematic structure cannot be relied on when considering online news stories, since it does not count for the free navigation (Zammit 2007) and the digital affordance represented by the contained clickable objects visualized in blue colour.

Such visualized blue attributes are structured within the reportage story to provide information through NYT internal articles or external websites. They then build up the negative tone of evaluation of the given reportage. As such, the body text of online reportage stories refutes the linear structure reading path of the upright pyramid. ledema's et al. (1994) orbital structure can be considered along with introducing some modifications to fit the genre of soft online news. Consider the following extract taken from the reportage under discussion:

\footnotetext{
CAIRO - Egyptians pile sugar into mugs of tea by the spoonful - or three or five. A staple long subsidized by the government for most of the population, sugar is the chief ingredient of the national pudding, Om Ali. It can feel like the only ingredient. It is also a prime reason that nearly a fifth of Egyptians have diabetes.

So a weekslong sugar shortage has plunged people into a panic. The sugar crisis, as it is known, has quickly become shorthand for the brewing anger against President Abdel Fattah el-Sisi's management of the economy and his overall rule.

"The people are going to snap," Ahmad el-Gebaly said as he turned away customers seeking sugar he did not have at his subsidized-goods store in Bulaq, a working-class neighborhood of Cairo.

"Nobody can stand him anymore," he added of Mr. Sisi. "Sugar is like rice and oil and wheat. You can never run out of it. You can never mess with it. Who can live without sugar?"

Still reeling from the political turbulence and militant attacks that followed the 2011 uprising, Egypt's economy is in free fall. Its pound is now worth 6 cents on the black market, about half its value a year ago.
}

The extract represents the delayed lead, the nutgraph and parts of the body development. The delayed lead takes the task of describing how sugar is an important staple to the Egyptians. The description offers a negative judgement tone evaluating the Egyptians' excessive consumptions of sugar, arguing that fifth of the Egyptians are diabetic. The main theme of the analyzed reportage is revealed in the second paragraph (the nutgraph). The nutgraph states the idea of prevailing sugar crisis along with Egyptians' reaction towards it.

A significant number of hyperlinks are embedded in the body text of the reportage under discussion. Considering such hyperlinks, it is found that they represent either internal or external contents. The internal content offers information on webpages that exist within the NYT site. As for the external content, it provides information on WebPages that exist outside of the NYT site. The following table illustrate the number of hyperlinks directed to internal content and hyperlinks to external webpages:

Table 1. Number of external and internal links

\section{Total number of embedded 8 hyperlinks}

\section{Number of internal contents}

\section{Number of external contents}

What is significant here is that the hyperlinks, whether internal or external carry the same negative evaluative stance against the current Egyptian government. The external links include social media web, citizengenerated blog, Al Ahram Egyptian newspaper and a report from the World Bank. All the external and internal links negatively evaluate the economic situation of Egypt while attributing it to president Abdel Fattah Al Sisi's government and its political practices. Orbitally, the nutgraph and the end are proposed to take the position of the nucli, whereas the delayed lead, body text, and the included clickable objects are moved to the satellite domain.

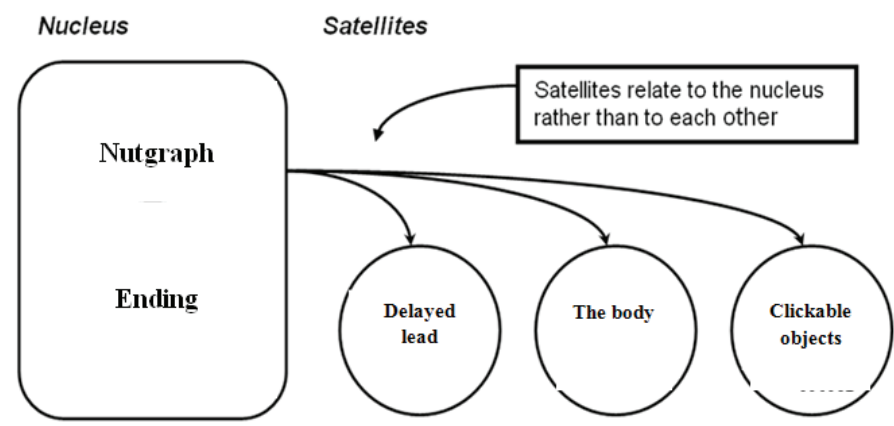

Figure 8. Proposed second nucleus-satellite unite 


\section{CONCLUSION}

Each online semiotic mode, verbal or non-verbal carries part of the evaluative stance. As such text loaded evaluative stance can be perceived as the production of the interplay between different semiotic resources. Studying text evaluative stance can be then considered within the parameter of genre and generic structure. Evaluative stance, as argued by the current study, is reflected, loaded and multiplied by generic structure affordances. The current study perceives text generic structure as semiotic instantiations of evaluative stance.

It follows the recent studies calling for a change in the theoretical and methodological approach when considering digital news due to the change in the semiotic potential afforded by the online medium. In this view, the study introduces a delicate refinement to ledema's et al (1994) and White's (1997) models for hard news structure so as to investigate the impact of the digital affordance of online news reportage on the intended evaluative stance. The study discusses the ways by which text generic structure influences the evaluative resources. The revised model is meant to trace the interaction between different semiotic systems as they are instantiated in texts.

In this respect, the study examines the evaluative stance embedded in the verbal-non-verbal intersemiosis involving the reportage nucleus-satellite elements. The analysis uncovers how the analyzed story evaluates the Egyptian recent sugar crisis along with negatively attributing it to the current government. It concludes that, NYT online reportages do not function within the tenet of the established upright pyramid, so often associated with English-language reportage stories. Online reportage significantly operates within the model of nucleus-satellite orbital structure after introducing some modifications to that model that suit the distinctive properties offered by the online domain.

As such, along with suggesting a new model to consider the generic structure of online reportage stories, this paper suggests that determining the generic structure of texts helps in the process of investigating evaluation instantiation. The study concludes that online reportage can be recognized as a macro genre that includes two interrelated yet independent sub-genres with two nucleus-satellites unites.

However, the suggestion for a revised genre model in the current study is not based on an extensive empirical study of various types of websites. The observation is restricted to a one online reportage story. A more empirical footing is needed and a large set of data are required to be able to make claims about online reportage stories schematic structure and its relation to the intended evaluative stance.

\section{BIBLIOGRAPHY}

Baldry, Antony and Paul Thibault. Multimodal Transcription and Text Analysis. London: Equinox Publishing, 2010.

Bateman, John A. Multimodality and Genre: A Foundation for the Systematic Analysis of Multimodal Documents. UK \& New York: Palgrave Macmillan, 2008.

Bateman, John. "Genre in the Age of Multimodality: Some Conceptual Refinements for Practical Analysis." Evolution in Genre: Emergence, Variation, Multimodality (2014): 237-269.

Bateman, John, Judy Delin and Renate Henschel. "Multimodality and Empiricism: Methodological Issues in the Study of Multimodal Meaningmaking". Perspectives on Modality (2002): 6589.

Bateman, John, Judy Delin and Renate Henschel. "Mapping the Multimodal Genres of Traditional and Electronic Newspapers". New Directions in the Analysis of Multimodal Discourse (2007): 147172.

Bateman, John A. and Veloso Francisco O.D. "The Semiotic Resources of Comics in Movie Adaptation: Ang Lee's Hulk (2003) as a Case Study". Studies in Comics (2013): 137-159.

Bhatia, Vijay K. Analyzing Genre: Language Use in Professional Settings. London, Longman, 1993. 
Bhatia, Vijay K. "Applied Genre Analysis: A Multiperspective Model". Ibérica: Revista de la Asociación Europea de Lenguas para Fines Específicos (AELFE) 4 (2002): 3-19.

Bhatia, Vijay K. "Towards Critical Genre Analysis." Advances in Discourse Studies (2008): 166-177.

Bhatia, Vijay K. "Critical Reflections on Genre Analysis". Ibérica, 24 (2012): 17-28.

Canavilhas, Joao. "Web Journalism: From the Inverted Pyramid to the Tumbled". Media and Arts Department University of Beira Interior Covilhã, (2013): 1-17.

Caple, Helen. "Intermodal Relations in Image-nuclear News Stories." Multimodal Semiotics: Functional Analysis in Contexts of Education (2008 a): 125138.

Caple, Helen. "Reconciling the Co-articulation of Meaning between Words and Pictures: Exploring Instantiation and Commitment in Image Nuclear News Stories". Questioning Linguistics (2008b): 77-94.

Caple, Helen. "Playing with Words and Pictures: Intersemiosis in a New Genre of News Reportage." PhD thesis, Australia: University of Sydney, 2009.

Caple, Helen. Photojournalism: A Social Semiotic Approach. Basingstoke: Palgrave Macmillan, 2013.

Chenguang, Chang. "English Idioms and Appraisal." System, Function, Appraisal (2007): 1-9.

Coffin, Caroline and Kieran O'Halloran. "Finding the Global Groove: Theorising and Analyzing Dynamic Reader Positioning Using Appraisal, Corpus and a Concordancer'. Journal of Critical Discourse Studies 2 (2012): 143-163.

Deuze, M. Epilogue: Towards a sociology of online news. In Paterson, C. \& Domingo, D. (Eds.) Making Online News, 199-210. New York: Peter Lang (2008).

Djonove, Emilia. "Analysing the Organisation of Information in Websites: From Hypermedia Design to Systemic Functional Hypermedia Discourse
Analysis". Published Doctoral Thesis, University of New South Wales: Sydney, 2005.

Djonov, Emilia. "Website Hierarchy and the Interaction between Content Organization, Webpage and Navigation Design: A Systemic Functional Hypermedia Discourse Analysis Perspective". Information Design Journal 2 (2007): 144-162.

Djonov, Emilia, Sumin Zhao and John S. Knox. "Interpreting Websites in Educational Contexts: A Social-semiotic, Multimodal approach". International Handbook of Interpretation in Educational Research (2015).

Du Bois, John, W. "The Stance Triangle". Stancetaking in Discourse: Subjectivity, Evaluation, Interaction (2007): 139- 182.

Economou, Dorothy. "Photos in the News: Appraisal Analysis of Visual Semiosis and Verbal-visual Intersemiosis". Published Doctoral Thesis, University of Sydney, 2009.

Englebretson, Robert. "Stance Taking in Discourse: An Introduction." In Stancetaking in Discourse: Subjectivity, Evaluation, Interaction, 1-25. Amsterdam: John Benjamins, 2007.

Fairclough, Norman. Discourse and Social Change. Cambridge: Polity Press, 1992.

Fairclough, Norman. Media Discourse. London: Edward Arnold, 1995.

Fairclough, Norman. Analyzing Discourse: Textual Analysis for Social Research. London: Routledge, 2003.

Feez, Susan, Rick ledema, Peter White and Helen De Silva Joyce (Eds.). Media Literacy. Sydeny: Surry Hills, 2008.

Gibson, James J. The Ecological Approach to Visual Perception. Hillsdale, NJ: Erlbaum, 1997.

Gill, Ann M. and Karen Whedbee. "Rhetoric". In: T. A. van Dijk. Discourse as Structure and Process (1997): 157-184.

Hiippala, Tuomo. "The Interface between Rhetoric and 
Layout in Multimodal Artefacts". Literary and Linguistic Computing 3 (2013): 461-471.

Hiippala, Tuomo. The Structure of Multimodal documents: An Empirical Approach. New York and London: Routledge, 2015.

Hiippala, Tuomo. (2017). "The Multimodality of Digital Longform Journalism". Digital Journalism 4 (2017): 420-442.

Hood, Susan. "Voice and Stance as Appraisal: Persuading and Positioning in Research Writing across Intellectual Fields". Stance and Voice in Written Academic Genres, edited by Guinda Carmen. Palgrave: Macmillan, 2012.

Johnston, Jane (2007). "Turning the Inverted Pyramid Upside Down: How Australian Print Media is Learning to Love the Narrative". Asia Pacific Media Educator, no. 1 (2007): 1-15.

Knox, John. "Visual-verbal Communication on Online Newspaper Home Pages." Visual Communication, no. 1 (2007): 19-53.

Knox, John. Multimodal Discourse on Online Newspaper Home Pages: A Social-semiotic Perspective. Australia: University of Sydney, 2009.

Kourdis, E \& Yoka, C. Intericonicity as Intersemiotic Translation in a Globalized Culture. In WANG \& Jl (eds.), Our World: A Kaleidoscopic Semiotic Network, Proceedings of the 11th World Congress of the IASS/AIS, 162 - 176. Hohai University Press, 2012.

Kress, Guther. "What is Mode?" In Handbook of Multimodal Analysis, edited by Carey Jewitt, 5467. Routledge, 2009.

Kress, Guther. Multimodality: A Social Semiotic Approach to Contemporary Communication. London and New York: Routledge, 2010.

Kress, Guther and Van Leeuwen T. Reading Images: The Grammar of Visual Design. London and New York: Routledg, 2006.

Kucher, Kostiantyn., Teri Schamp-Bjerede, Andreas
Kerren, Carita Paradis and Magnus Sahlgren "Visual Analysis of Online Social Media to Open up the Investigation of Stance Phenomena." Information Visualization 2 (2016): 93-116.

ledema, Rick. "Multimodality, Resemiotization: Extending the Analysis of Discourse as Multi-semiotic Practice." Journal of Visual Communication 2 (2003): 29-57.

Lemke, Jay. "Multiplying meaning: Visual and verbal semiotics in scientific text." In Reading science: Critical and Functional Perspectives on Discourses of Science, edited by Martin, J. R. and Robert Veel, 87-113. London and New York: Routledge, 1998.

Ljung, Magnus. (2000). "Newspaper Genres and Newspaper English." In English Media Texts: Past and Present, Language and Textual Structure, edited by Fredrick Ungerer, 131-149. Amsterdam: John Benjamins Publishing Company, 2000.

Makki, Mohamed and PRRR White. "Socio-cultural Conditioning of Style and Structure in Journalistic Discourse: The Distinctively "objective" Textuality of Iranian Political News Reporting." Discourse, Context and Media 21 (2018): 54-63.

Mann, W.C. and Sandra Thompson. "Rhetorical Structure Theory: Toward a Functional Theory of Text Organization." Text 8 (1988): 243-81.

Martinec, Radan and Andrew Salway. "A System for Image-text Relations in New (and old) Media." Visual Communication 4 (2005): 337-371.

Martin, James. "Genre, Ideology and Intertextuality: A Systemic-functional Perspective." Linguistics and the Human Sciences 2 (2007): 275-298.

Keynote: Exploring Content: Building knowledge in school discourse. Madrid: Teacher Learning for European Literacy Education, 2013.

Genre Relations -Mapping Cultures. London: Equinox, 2008.

Miller, Carolyn R. "Genre as Social Action". In: A. Freedman, A. \& Medway." In Genre and the New 
Rhetoric, edited by Aviva Freedman and Peter Medway, 23-42. London: Taylor \& Francis Ltd, 1994.

Kay L. O'Halloran, Sabine Tan \& Peter Wignell. Intersemiotic Translation as Resemiotisation: A Multimodal Perspective. Signata [Online]: 2016

Royce, Terry D. "Intersemiotic Complementarity: A Framework for Multimodal Discourse Analysis". In New Directions in the Analysis of Multimodal Discourse, edited by Terry D. Royce, T. and Wendy Bowcher, 63-109. New York: Routledge, 2007.

Serafini, Frank. "Reading Multimodal Texts: Perceptual, Structural and Ideological Perspectives". Children's Literature in Education 41, (2010): 85104.

Song, Yang. "A Genre-aware Approach to Online Journalism Education". Procedia - Social and Behavioral Sciences 51 (2012): 400-404.

Steensen, Steen. "Online Feature Journalism: A Clash of Discourses." Journalism Practice 3 (2009): 13-29.

Swales, John. Genre Analysis: English in Academic and Research Settings. Cambridge: Cambridge University Press, 1990.

Tan, Sabine. "Modelling Engagement in a Web-based Advertising Campaign." Visual Communication 91, (2008): 91-115.

Tardy, C. M. "Genre analysis". In Continuum Companion to Discourse Analysis, edited by Ken Hyland and
Paltridge, 54-68. London: Continuum, 2011.

Thibault, Paul J. "Multimodality and the school science textbooks." In Corpora testuali per ricerca, traduzione e apprendimento linguistic, edited by Torsello, C. T., Brunetti, and Penell: 293-335. Padova: Unipress, 2001.

Thompson, Geoff and Susan Hunston. "Evaluation: An introduction." In Evaluation in text: Authorial Stance and the Construction of Discourse, edited by Susan Hunston and Thompson, Geoff, 1-27. New York: Oxford University Press, 2000.

White, Peter R.R. "Death, disruption and the moral order: The narrative impulse in mass-media hard news reporting". In Genres and Institutions: Social Processes in the Workplace and School, edited by Francis Christie and J.R Martin, 101-133. London: Cassell, 1997.

Zhang, Yiqiong and Kay L. O'Halloran. "Toward a Global Knowledge Enterprise: University Websites as Portals to the Ongoing Marketization of Higher Education." Critical Discourse Studies 10 (2013): 468-485.

Zammit, Katina. "Popular Culture in the Classroom: Interpreting and Creating Multimodal Texts". In Advances in Language and Education, edited by McCabe, A., O'Donnell, M., and Whittaker, R., 61-76. New York: Continuum, 2007. 